\title{
Research on the Applications of Molecular Biotechnology in Medicine
}

\author{
Lan Wang, Fang Wang
}

College of Chemical Engineering and Food Technology, Zhongzhou University, Zhengzhou, He'nan, China

lanwang@163.com

Keywords: Molecular biotechnology, Western medicine, Traditional Chinese medicine.

\begin{abstract}
In recent decades, molecular biological technology has gradually applied in western medicine and traditional Chinese Medicine. This paper firstly introduces the concept of molecular biological technology, and expounds the applications in the diagnosis and treatment of diseases in western medicine and the applications in mechanism and development of traditional Chinese medicine in order to provide references for the related researchers.

People gradually realize that the essence of health and disease problems ultimately can be attributed to the interactions between biomolecules since the new century. So, in recent decades, people have enhanced the study of molecular biological technology in the applications in medicine. Molecular biology technology plays an important role in the medical applications. For example, in some genes involved in the application of all cannot do without the molecular biological technique. At the same time in the occurrence of disease form mechanism and disease diagnosis and drug is unavoidable in the development of molecular biological technology. The development of these technologies will also accelerate the development of molecular biological technology itself, making the technology and medicine into a spiral development and laying a solid theoretical basis for western medicine and traditional Chinese medicine.
\end{abstract}

\section{Concept of Molecular Biotechnology}

Molecular Biotechnology is a technical which achieves the transformation of the structure of biological people get products people need to use proteins and nucleic acids properties through the study of proteins, nucleic acids in molecular level and molecular biological technology, to grasp its nature. The main molecular biotechnology includes the known gene synthesis, unknown sequence synthesis, Southern blot, Northern blot hybridization. Molecular biological technology is mainly used for recombinant protein production, gene transformation of species, gene therapy, gene transformation and criminal judge, environmental protection etc. Molecular biotechnology is the cell fusion and gene recombination technology as the foundation, through study of some characteristics and functions of organism itself, the new design and construct a new species with human intervention or new lines, and then to carry out mutual production and processing combined with some engineering principles, a comprehensive technological system to provide additional goods and services. So we often put the molecular biological technique called biological engineering, it also marks the main research direction of modern biological technology. In these research direction, cell engineering, genetic engineering, enzyme engineering, research on the sequencing and DNA chip technology is a main research direction. The study of lymphocyte hybridism and DNA recombination made the birth of molecular biological technology in 1970s. After 40 years of development, molecular biological technology has become the mainstream direction of research of modern medicine. Practice has proved that the molecular biology technology to solve human health, food, energy and environment of some related to human development have broad application prospects. Therefore, molecular biology techniques have been brought from all aspects of attention has become a technology to businesses and governments are very concerned about. Researchers strengthen the research on this aspect and investment afterwards. The twenty-first century will be an era of the rapid development of molecular biology techniques, and the application of molecular biology technology in medicine will also appear blowout type development prospects. The developments of the past several decades make us believe that the 
applications of molecular techniques will bring new development opportunities for modern medicine.

\section{Applications in Western Medicine}

Applications in Diagnosis of Diseases. The outbreaks of large scale diseases have occurred in our country several times such as the H1N1 influenza and H7N9 of avian influenza type, which caused a certain degree panic in society. On one hand, this is because the disease has its own characteristics; on the other hand it is due to the lack of fast and effective detection means. In other words, these viruses are so small that we can't use the general method cannot distinguish. The technology of PCR (Polymerase Chain Reaction) is a method of in vitro enzymatic synthesis of specific DNA fragment, with just a few samples to the number needed in analysis of DNA quickly expanded to millions of times, thus the diagnosis of pathogenic microorganisms. In this process, the PCR technology as a magnifying glass, so that immediately appeared pathogenic microorganism tiny. Of course, this is just one application of them. The technique is also used for prenatal diagnosis. The technical development of the PCR oncogene detection and diagnosis and the paternity testing quickly, especially the real-time fluorescence quantitative PCR, can more quickly get accurate test results showed that, by using real time fluorescent quantitative PCR method to detect human cytomegalovirus infection in patients with bone marrow transplantation is more sensitive than the conventional methods. In addition, the method can also be used to HIV, hepatitis $\mathrm{B}$, hepatitis $\mathrm{C}$ virus, human papilloma viral pathogens of accurate detection. The expensive equipment, complex operation and higher environmental requirements restrict the application of this method. With the miniaturization and portable of PCR instrument, the instrument will not only exist in the university laboratory, but also can appear in the people's life. Molecular biological sensor is the use of certain biological or chemical fixation technology, the biological recognition element (such as enzyme, antibody, antigen, protein, nucleic acid, receptors, cell, microbial, plant and animal tissue) is fixed on the transducer, when the object to be measured and the biological recognition element specificity after reaction, the generated by the transducer the reaction results can be transformed into output, the detection of electrical and optical signals, thus tested material qualitative and quantitative analysis, so as to achieve the purpose of detection and analysis. Detection of molecular biological sensor can be widely applied to a variety of substances, trace protein of body fluid in small organic molecules, nucleic acid etc. These projects are an important basis for the analysis of clinical diagnosis and disease.

Applications in Treatment of Diseases. Several successful cases which used gene therapy have appeared in the USA since 1990s. Take the 4 year old Schilder for example. The genetic lack of a correlation with the innate immune function of the enzyme led him loss of immune function and can only live in sterile isolation tent. Scientists will her lack of enzyme gene by gene therapy into its genome, make up the genetic defect, after treatment, the immune function is becoming perfect, eventually lead a normal life. In 1995, the world has more than 100 gene therapy clinical plan, by 2004 this data has been increased to 987. After doctors' and scientists' study on the key basic problem in gene therapy as well as the government strengthen the supervision, gene therapy will be more standardized and rational. Clinical programs increasingly show that has a good application prospect in gene therapy. Basic research and clinical trials and the international parallel to our country in the gene therapy of hemophilia,, single gene genetic disease, malignant tumor, cardiovascular disease carried out the research of gene therapy of basic and clinical trials, and the establishment of a national plan of biological field of viral vectors in gene research and development base, mainly the development of adenoma-associated virus vector development and industrialization. Stem cells are progenitor of human tissue cells. In other words, these cells can differentiate into all kinds of human organs due to various reasons. Some patients with organ failure, also is the key "parts" is bad, need to be replaced, transplantation of others or other animal organs, all possible rejection of these "parts". Using molecular biology techniques, the patients were cells into stem cells, and the differentiation of stem cells into the corresponding tissues or organs, so as to replace failure parts of body "'". The first British 3D prints development to human embryonic stem 
cells, and make the printing of the embryo. Stem cells still maintain the cell activity, which indicates that we can custom our organs like our clothes in the near future.

\section{Applications in Traditional Chinese Medicine}

Applications in Mechanism of Traditional Chinese Medicine. With the rapid development of our economy, the research technique and the method of traditional Chinese medicine has also been improved. The development and application of molecular biology technology has promoted the development of life science. The traditional Chinese medicine research has widely used in the study of syndrome and the molecular biology in recent years. This will be a good mechanism, basic theory, from the molecular level to study and explore traditional Chinese medicine Chinese medicine in prevention and treatment of related diseases, promote the combination between traditional Chinese medicine and Western medicine. Traditional Chinese medicine is the first-line drug use more clinically, patients with after the administration of drugs to direct lesions, effectively alleviate the condition but the medical profession for the mechanism of action of traditional Chinese medicine is not completely known, with the continuous application of molecular biology technology in the research of Chinese medicine, can effectively explore the mechanism of traditional Chinese medicine in patients after taking Chinese medicines, Chinese herbal medicine for links and links between the molecules of life exist more or less, when Chinese medicine play a role is bound to lead to the change of genetic information, and decided to change its basis is gene. According to the recent study results show, the expression and regulation of gene effects of bioactive components of Chinese medicine. Our researchers have experimented on ginseng, Radix Astragalin, epicedium and glossy as drug regulation in human cells in the body's immune function etc.. To promote the study of icariin showed the expression of IL-3m RNA and IL-6mRNA of mouse spleen cells, patients after taking Chinese medicines, drug can effectively induce lymphocyte IL-3mRNAIL-6mRNA genetic gene, and the relationship between promoting effect of patients with drug and drug dose closely. Using semi quantitative reverse transcription polymerase chain reaction (PT-PCR) method for determination of myocardial N-rasm RNA in rats of each group, found that ras oncogene in the formation of myocardial hypertrophy plays a certain role in the development process, the Chinese medicine of promoting blood circulation and eliminating phlegm therapy can reverse cardiac hypertrophy, its therapeutic mechanism may be inhibiting the high expression of myocardial ras proto oncogene in recent years using the gene chip technology to the traditional Chinese medicine compound was studied, using the traditional method of previous research is only limited to the study of function of one aspect or aspects of the compound traditional Chinese medicine, it is difficult to have a comprehensive understanding of the compound, especially to understand and develop the new function. Application of gene chip technology can quickly diagnoses the compound effects on expression of some genes in the genome.

Applications in Development of Traditional Chinese Medicine. With the rapid development of medical technology in our country, the technology of molecular biology has made outstanding contributions in traditional Chinese medicine in in the recent years. Many wild medicinal resources are on the verge of extinction in China. Moreover, a lot of artificial cultivation and the traditional Chinese medicine quality are not high, making the clinical use of Chinese patent medicine very difficult. The clinical use of medicinal plant transgenic technology able to seed breeding of related plants, effectively improves the quality of medicines, and can obtain the mutant ability stronger compounds. The biotransformation application of plant cell culture technology of digitalis culture cell, screening high-yield cell and obtained a quite good genetic stability, also has been training the active anticancer constituents three sharp cedar ester content higher than three sharp cedar primary cultures of plant cells. The study found strongly inhibited the HIV virus and many other viruses, has significant clinical effect, the increasing demand. Now, its coding gene has been cloned and highly expressed. The development of molecular biology techniques make use of to obtain transgenic animal like musk, bezoar, rhinoceros horn and some animal medicine become possible, thus greatly saving resources, protecting the endangered animal. With the rapid development of medical technology, gene research in China has entered the post genome era, after the study of traditional 
Chinese medicine syndromes in the genomic era, with the gene structure and function research breakthrough, pathogenic gene discovery. One of the research targets for drugs is to speculate the screening of drug action through the differential expression gene target cells which were compared before and after drug treatment so as to find a guide drug.

\section{Conclusion}

This paper analyzes the applications of molecular biology technology in western medicine and traditional Chinese medicine. Throughout the development of modern medical molecular biological technology and its industry, the prospects are bright. Experts predict that, with the human genome project process development, modern biological technology will bring about the rapid development of modern medicine which can change the life of human beings radically.

\section{References}

[1] George Poste, Molecular medicine and information-based targeted healthcare. Nature Biotechnology. 19 - 21 (2008).

[2] Ruth Freitag, An Introduction to Molecular Biotechnology, J. Biotechnology Journal. 27 - 31 (2008)

[3] Q. Song, J. Peng, C.K.Tseng, Molecular biotechnology of marine algae in China, third ed., Springer Netherlands, Berlin, 2004. 